\title{
La investigación científica en los estudiantes de grado y posgrado
}

Lesbia Jeannette Buitrago Reyes ${ }^{1}$

\section{RESUMEN}

En este artículo se describe la participación de los estudiantes de grado y posgrado de la Universidad Nacional Autónoma de Honduras en proyectos de investigación científica en el período 2007-2015, por medio de fondos concursables que impulsa la UNAH con las convocatorias de becas de investigación. Se identifican las unidades académicas con mayor o menor participación así como las fortalezas y debilidades que presentan los proyectos que concursan y los porcentajes de aprobación o no de estas propuestas.

\section{INTRODUCCIÓN}

Por medio de la investigación científica y tecnológica se busca interpretar y dar explicación a los fenómenos de la realidad. La ciencia permite someter estas interpretaciones a comprobación mediante una sistematización para lograr objetividad en las relaciones que se establecen en dichas comprobaciones.

La Universidad Nacional Autónoma de Honduras (UNAH), mediante el Plan Táctico de la Reforma Universitaria, plantea como programa prioritario "la promoción e inversión en investigación, gestión del conocimiento y propuestas de solución a los problemas nacionales". En tal sentido, ha establecido una política institucional que brinda directrices en materia de investigación, desarrollo tecnológico e innovación para el quinquenio 2015-2019" (Dirección de Investigación Científica, 2015 ).

Nos acercamos a cumplir un siglo desde la reforma de Córdoba (1918), donde la investigación además de la docencia y la vinculación se incluyen como funciones básicas de la universidad, las que no son estáticas ni independientes entre sí, sino

1 Jefa del departamento Proyectos de Investigación, DICYP, UNAH: investigacionunah.proyectos. lb@gmail.com 
que forman parte de un sistema dinámico, interdependiente que permite que las unas enriquezcan a las otras.

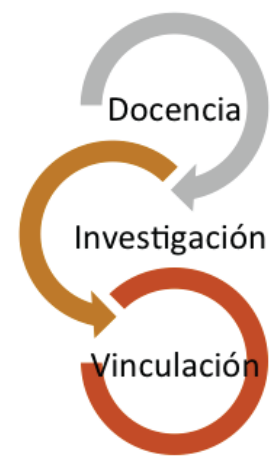

Funciones básicas de la Universidad

La docencia transmite el conocimiento, la investigación lo genera y la vinculación lo aplica. En esta dinámica la docencia es enriquecida por la investigación. El conocimiento es transferido a la sociedad por medio de la vinculación; y regresa de nuevo con nuevas interrogantes que estimulan a la investigación, la cual a su vez -como ya se indicó- enriquece la docencia.

En el caso de los estudiantes, en esta dinámica deben desarrollar competencias investigativas, entendidas como "el conjunto de conocimientos, habilidades y actitudes que se aplican en el desempeño de una función productiva o académica... tomando todas sus dimensiones epistemológica, metodológica, técnica y social” (Maldonado, 2007).

Es necesaria la participación de los estudiantes de las universidades en la realización de investigaciones, pues con ello contribuyen al desarrollo científico, tecnológico y de innovación de la sociedad. "La acción investigativa estimula al estudiante a conceptualizar o categorizar para interpretar la realidad del contexto desarrollando teorías o modelos. Identificar los principios y normas que rigen la actividad investigativa, formativa o propiamente dicha. Permite reportar a la sociedad y a la comunidad académica, el conjunto de conceptos, ideas, razones, descripciones e interpretaciones que desde diferentes teorías y disciplinas que conforman la acción investigativa y construir permanentemente las formas de organización del quehacer investigativo, así como los principales modos de comunicación e interacción entre los grupos de investigación o investigadores y la comunidad académica o productiva, promulgando así la visibilidad de los investigadores o grupos" (Maldonado, 2007).

A los estudiantes que investigan -además de formarse profesionalmente- se les facilita desarrollar el pensamiento crítico por el hecho de observar, preguntar, experimen- 
tar e interpretar los fenómenos y contribuir a la solución de los problemas de la sociedad, al ser capaces de analizar problemas, buscar soluciones y aplicarlos.

\section{Apoyo de la UNAH a la formación investigativa de sus estudiantes}

La Dirección de Investigación Científica y Posgrado, desde el año 2007 ha impulsado el desarrollo de capacidades investigativas con cursos y diplomados en investigación científica, tecnológica y de innovación dirigidos a los profesores para que a su vez sean efecto multiplicador con sus estudiantes, así como la participación de estudiantes de grado y posgrado y profesores en el desarrollo de investigaciones mediante el concurso de becas de investigación.

El proceso de concursos inicia con la presentación del proyecto de investigación, cumplimiento de requisitos, evaluaciones metodológicas y financieras y notificación oficial tanto a los investigadores con proyectos aprobados como a quienes no se les ha aprobado, teniendo los últimos la oportunidad de una nueva presentación del proyecto en la siguiente convocatoria al incorporar las observaciones que se les dan.

La política de investigación científica, desarrollo tecnológico e innovación de la UNAH en el período 2015-2019 establece cinco ejes orientadores: a) Fomento de la investigación; b) Publicación, difusión y comunicación; c) Protección de los resultados de investigación; d) Capacitación en investigación y en gestión de la investigación y e) Gestión de la investigación. El eje de fomento de la investigación incluye el otorgamiento de becas de investigación mediante un fondo concursable, cuya convocatoria es pública y dos veces por año. Los tipos de becas son para participación de profesores y/o estudiantes, y se clasifican en: becas sustantivas ${ }^{2}$, becas básicas ${ }^{3}$, becas para formación de investigadores en temas prioritarios ${ }^{4}$, becas para docentes egresados de posgrado ${ }^{5}$, becas para estudiantes de posgrado y estudiantes de grado. Estas últimas se otorgan a estudiantes de la UNAH con excelencia académica, que habiendo cursado más del $80 \%$ de su respectivo plan de estudios presentan un proyecto de investigación de alta calidad y pertinencia, avalado por un profesor universitario y que responde a las prioridades de investigación de la universidad y de

\footnotetext{
${ }^{2}$ Son otorgadas a profesores para desarrollar proyectos inter o multidisciplinarios de investigación por un mínimo de dos profesores investigadores activos.

${ }^{3}$ Para el desarrollo de proyectos de investigación por un máximo de tres profesores investigadores, esta beca permite además realizar investigación como asignación académica.

${ }^{4}$ Son proyectos de Investigación para potenciar y fortalecer las capacidades investigativas de profesores con poca o ninguna experiencia en Investigación.

${ }^{5}$ Para la finalización de tesis o trabajo de graduación de posgrado
} 
la unidad académica a la que se encuentra adscrito. Las últimas tienen un monto de hasta L.25, 000.00 para estudiantes de grado y hasta L.40, 000.00 para estudiantes de posgrado (Dirección de Investigación Científica, 2015).

Este artículo se centra en las últimas, que -como ya se indicó- se destinan a estudiantes de grado y posgrado, para el desarrollo de proyectos de investigación, aunque al principio del mismo se expone la situación general de todas las becas.

\section{Participación en los concursos de Investigación}

En el cuadro 1 se presenta una estimación de la relación de participación de los estudiantes de grado y posgrado que postularon para obtener beca de investigación con respecto al promedio ${ }^{6}$ de estudiantes matriculados en su último año en la UNAH, en los períodos de convocatoria a becas de investigación. Ahí se observa una tendencia a mayor participación de un año al siguiente en grado: participaron 0.6 estudiantes por cada mil matriculados en 2014 , al siguiente año subió a 0.8 por cada mil matriculados y en el año 2016 fue de 1.9 postulantes por cada mil matriculados; para hacer un promedio durante el período de 1.1 estudiantes por cada mil matriculados. En el caso de los estudiantes de posgrado la participación -como se esperaría- es mayor: participaron 3.0 estudiantes por 100 matriculados en el año 2014, casi 2 por 100 matriculados en 2015 y un poco más de 2 estudiantes por cada 100 matriculados en 2016, para hacer un total de 2.4 participantes en los concursos por cada 100 estudiantes de posgrado matriculados en el período 2014-2016.

\section{Cuadro 1. UNAH 2014-2016: Relación de becas de estudiantes de grado y posgra- do postuladas con relación al promedio de su respectiva matrícula en último año}

\begin{tabular}{|c|c|c|c|c|c|c|}
\hline & \multicolumn{3}{|c|}{ Becas de estudiantes de grado } & \multicolumn{3}{c|}{ Becas de estudiantes de posgrado } \\
\cline { 2 - 7 } Año & $\begin{array}{c}\text { Matrícula } \\
\text { último año }\end{array}$ & $\begin{array}{c}\text { Número } \\
\text { de postulaciones }\end{array}$ & $\begin{array}{c}\text { Participación } \\
\text { (por mil) }\end{array}$ & Matrícula & $\begin{array}{c}\text { Número de } \\
\text { postulaciones }\end{array}$ & $\begin{array}{c}\text { Participación } \\
\text { (por cien) }\end{array}$ \\
\hline 2014 & 9,614 & 6 & 0.6 & 1,281 & 39 & 3.0 \\
2015 & 9,614 & 8 & 0.8 & 1,057 & 20 & 1.9 \\
2016 & 9,614 & 18 & 1.9 & 1,065 & 22 & 2.1 \\
Total & 28,842 & 32 & 1.1 & 3,403 & 81 & 2.4 \\
\hline
\end{tabular}

Fuente: Secretaría Ejecutiva de Desarrollo Institucional, UNAH 2016

\footnotetext{
${ }^{6}$ Por la falta de información, para hacer las estimaciones de participación en el cuadro 1 se han tomado los datos de los estudiantes de medicina de último año, de la tercera y cuarta cohorte de 2016, extrapolándolo a las 14 facultades y 9 centros regionales presentando un aproximado.
} 
Si bien son estrictos los requisitos de participación para los estudiantes -especialmente que sean de último año y de excelencia académica-, es importante que las unidades académicas estimulen a sus alumnos a aplicar en mayor medida a las becas de investigación, como una forma de completar su formación académica -por un parte- y también de cimentar en ellos el pensamiento crítico, sus competencias investigativas y su innovación; lo que a nivel macro contribuirá a hacer un país más competitivo.

\section{Número de becas otorgadas}

El gráfico 1 presenta el total de becas de investigación otorgadas a profesores y estudiantes desde el año 2007 hasta el 2015, alcanzando 233 becas de diferentes tipos, incluyendo 14 becas a estudiantes de grado y 48 a estudiantes de posgrado, totalizando éstas $26.6 \%$ del total de becas otorgadas en dicho período: $6 \%$ a estudiantes de grado y $20.6 \%$ a los de posgrado.

\section{Gráfico 1. UNAH 2007-2015: becas otorgadas según su tipo}

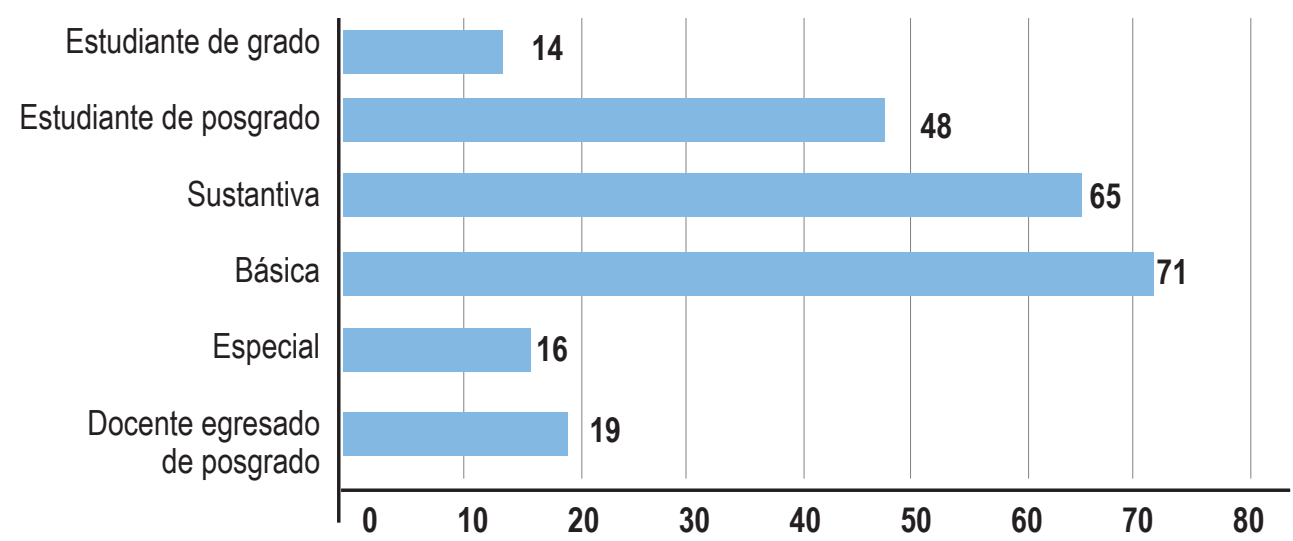

Fuente: La investigación científica en la UNAH, Todo en cifras 2007-2015.

El gráfico 2 muestra la inversión según tipo de beca. Las destinadas a estudiantes de grado y posgrado totalizan L. $1,724,090.00$ representando $4.3 \%$ del total de los fondos otorgados a becas de investigación en el período ya indicado $(0.9 \%$ para estudiantes de grado y $3.4 \%$ para los de posgrado). ${ }^{7}$ Las becas para estudiantes de grado tienen un monto máximo de L. 25,000.00 mientras que las destinadas a estudiantes de posgrado llegan hasta L. 40,000.00.

\footnotetext{
${ }^{7} 76.7 \%$ fueron otorgadas a las becas sustantivas, lo cual era de esperarse pues una beca sustantiva puede recibir hasta L.500, 000.00 .
} 
Gráfico 2. UNAH 2007-2015: Inversión en becas de investigación científica según tipo y presupuesto

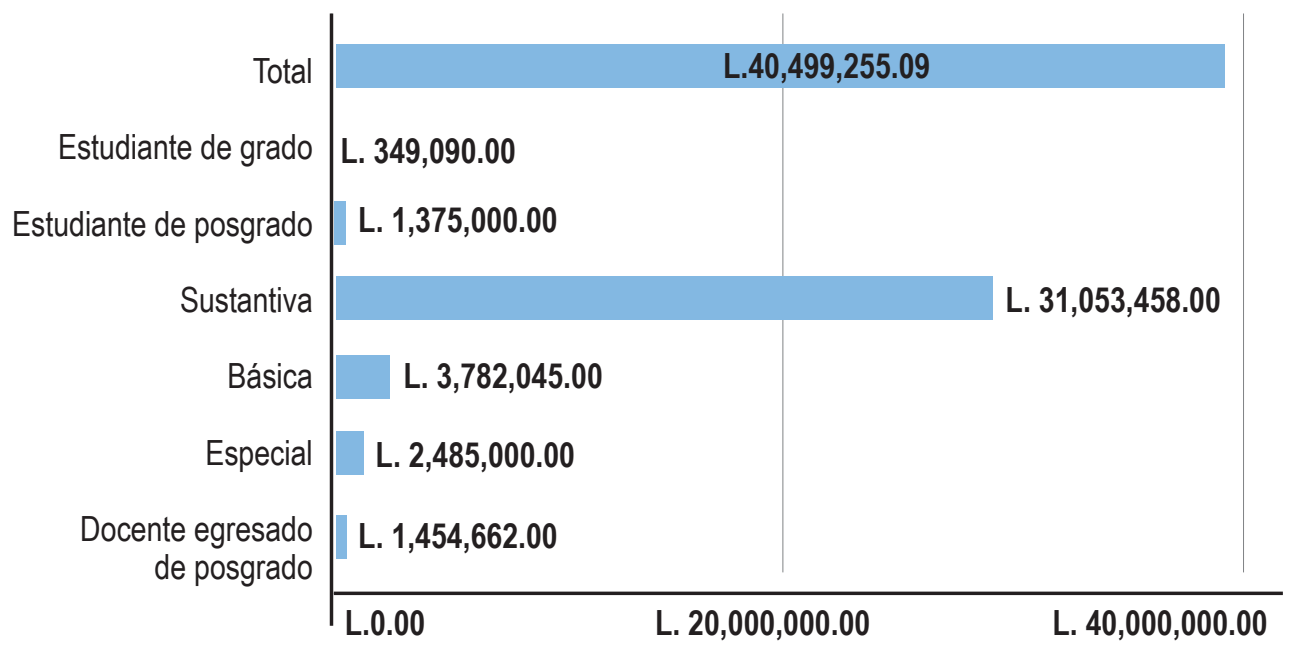

Fuente: La investigación científica en la UNAH, Todo en cifras 2007-2015.

Becas otorgadas a estudiantes de grado, posgrado y para tesis de posgrado por Facultades

El cuadro a continuación presenta la participación en becas de investigación de los estudiantes de grado y posgrado por Facultades y Centros Regionales de la UNAH. Ahí se observa que el mayor número fue recibido por estudiantes de posgrado con 48 becas, constituyendo $77.4 \%$ del total de becas concedidas a estudiantes entre los años 2007 y 2015; y 79.8\% del monto otorgado; mientras que las de grado han constituido $17.3 \%$ del mismo total con 14 becas con monto total de L1, 724, 090.00 de la participación de todas las facultades. 
Cuadro 2. UNAH 2007-2015: Cantidad y monto de becas otorgadas a estudiantes de grado y estudiantes de posgrado y profesores egresados de posgrado por Facultad y Centro Regional

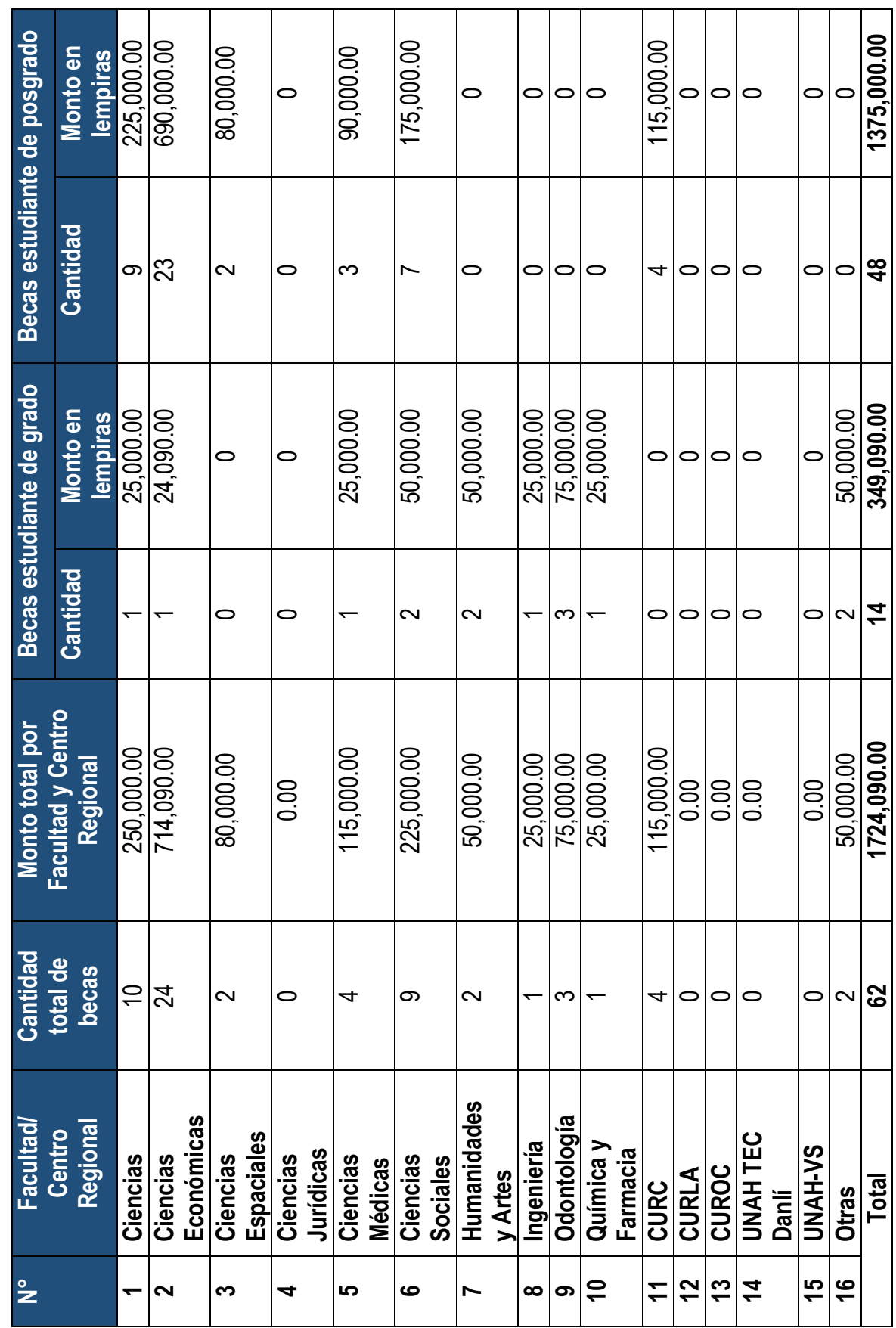

Fuente: La investigación científica en la UNAH, Todo en cifras 2007-2015. 
Al analizar sólo las becas de estudiantes de grado y posgrado puede observarse que la mayor participación ocurre en la facultad de Ciencias Económicas, con un total de 24 becas que concentraron $41.4 \%$ del monto total utilizado para estudiantes en todas las facultades; seguida de Ciencias con 10 becas, con 14.5\% del monto total; Ciencias Sociales con 9 becas, con monto del 13.1\%, Ciencias Médicas y CURC -único centro regional con participación en estos tipos de becas- con 4 becas cada una alcanzando el $6.7 \%$ del monto total en cada una de esas unidades académicas; Odontología con 3 becas, con monto del $4.4 \%$, Humanidades y Artes y Ciencias Espaciales con 2 becas cada una, pero con monto del $2.9 \%$ la primera y $4.6 \%$ la segunda ${ }^{8}$; Química y Farmacia e Ingeniería con 1 beca cada una, con monto de 1.5\% en cada facultad, siempre con respecto al monto total.

Si se agrega la participación de profesores con becas de docentes egresados de posgrado -utilizadas para hacer tesis de graduación en el posgrado que cursa -el número de becas pasa de 62 a 81 becas y el monto casi se duplica, al pasar a $\mathrm{L}$ $3,178,752.00$. Por facultades se observa nuevamente en primer lugar a la Facultad de Ciencias Económicas, con 26 becas y el $27.5 \%$ del monto total; seguida por la de Ciencias con 15 becas y con $19.4 \%$ del monto total; Ciencias Sociales con 12 becas que concentraron 14.6\%; Ciencias Médicas con 6 becas que obtuvieron $8.7 \%$ del monto; Odontología y CURC con 4 becas cada uno, la primera concentrando $4.9 \%$ del presupuesto y el segundo el $3.6 \%,{ }^{9}$ Ciencias Espaciales con 3 becas con monto del $5.0 \%$ del monto total; la facultad de Humanidades y Artes con 2 becas y concentrando 1.6\% del monto; y Química y Farmacia, CUROC, UNAH TEC-Danlí e Ingeniería con 1 beca cada uno ${ }^{10}$, concentrando los siguientes porcentajes del monto total: CUROC con 2.5\%, UNAH TEC-Danlí con 1.6\%, Química y Farmacia e Ingeniería con $0.8 \%$ cada uno (ver Cuadro 3 ).

\section{Número de becas aprobadas vs postuladas}

Al analizar el porcentaje de participación de los estudiantes (becas postuladas) con respecto al total general de becas, en el cuadro 4 se observa que en 2014 fue $38.8 \%$, en 2015 del $29.5 \%$ y en 2016 de $35.4 \%$. En el conjunto del período $2014-2016$ el porcentaje de becas aprobadas con respecto a las postuladas por el total de estudiantes de grado y posgrado fue de $34.9 \%$; es decir que un poco más de la tercera parte de las becas postuladas fueron de estudiantes de grado y posgrado.

\footnotetext{
${ }^{8}$ Aunque las dos presentan número igual de becas los montos son diferentes por ser tipos de becas diferentes que tienen montos diferentes.

${ }^{9}$ Idem anterior.

10 Ídem anterior
} 
Cuadro 3. UNAH 2007-2015: Cantidad y monto de becas otorgadas a estudiantes de grado, estudiantes de posgrado y profesores egresandos de posgrado por Facultad y Centro Regional

\begin{tabular}{|c|c|c|c|c|c|c|c|c|c|c|c|c|c|c|c|c|c|c|}
\hline \multirow{2}{*}{ 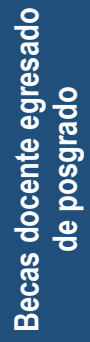 } & $\begin{array}{l}\text { 등 옳 } \\
\text { 올 흘 } \\
\text { 을 은 }\end{array}$ & 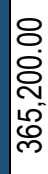 & $\begin{array}{l}8 \\
8 \\
8 \\
8 \\
0\end{array}$ & $\begin{array}{l}8 \\
8 \\
8 \\
8 \\
\varnothing\end{array}$ & 0 & $\begin{array}{l}8 \\
8 \\
8 \\
8 \\
8\end{array}$ & $\begin{array}{l}8 \\
8 \\
8 \\
8 \\
\infty 0 \\
\text { ஸे }\end{array}$ & 0 & 0 & $\mid \begin{array}{l}8 \\
8 \\
8 \\
8 \\
0 \\
\varnothing\end{array}$ & 0 & 0 & 0 & $\begin{array}{l}8 \\
8 \\
8 \\
8 \\
0 \\
0 \\
\infty\end{array}$ & 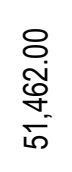 & 0 & 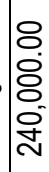 & 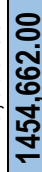 \\
\hline & 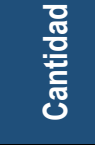 & ما & $\sim$ & $\leftarrow$ & 0 & $\sim$ & $m$ & 0 & 0 & - & 0 & 0 & 0 & - & $\leftarrow$ & 0 & $m$ & $\stackrel{2}{\square}$ \\
\hline \multirow{2}{*}{ 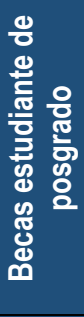 } & $\begin{array}{l}\text { 등 온 } \\
\text { 을 흥 } \\
\text { 을 흔 }\end{array}$ & 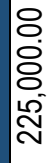 & $\begin{array}{l}8 \\
\ddot{8} \\
8 \\
8 \\
8\end{array}$ & $\begin{array}{l}8 \\
8 \\
8 \\
8 \\
\varnothing\end{array}$ & 0 & $\begin{array}{l}8 \\
8 \\
8 \\
8\end{array}$ & $\begin{array}{l}8 \\
8 \\
8 \\
0 \\
\stackrel{5}{\circ}\end{array}$ & 0 & 0 & 0 & 0 & $\begin{array}{l}8 \\
8 \\
8 \\
0 \\
10\end{array}$ & 0 & 0 & 0 & 0 & 0 & 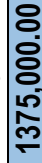 \\
\hline & 웡 & $\sigma$ & $\widetilde{N}$ & $\sim$ & 0 & $m$ & N & 0 & 0 & 0 & 0 & + & 0 & 0 & 0 & 0 & 0 & $\stackrel{\infty}{q}$ \\
\hline \multirow{2}{*}{ 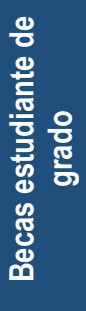 } & $\begin{array}{l}\text { 덩 연 } \\
\text { 을 흥 } \\
\text { 을 흔 }\end{array}$ & $\begin{array}{l}8 \\
8 \\
8 \\
8 \\
\text { ஸे }\end{array}$ & $\begin{array}{l}8 \\
\circ \\
\text { Oे } \\
\text { i }\end{array}$ & 0 & 0 & $\begin{array}{l}8 \\
8 \\
8 \\
\text { एँ }\end{array}$ & $\begin{array}{l}8 \\
8 \\
8 \\
8 \\
\circ\end{array}$ & $\begin{array}{l}8 \\
8 \\
8 \\
8 \\
\circ \\
\circ\end{array}$ & $\begin{array}{l}8 \\
8 \\
8 \\
8 \\
\text { స్. }\end{array}$ & $\begin{array}{l}8 \\
8 \\
8 \\
8 \\
0 \\
1\end{array}$ & $\begin{array}{l}8 \\
8 \\
8 \\
\text { ¿ }\end{array}$ & 0 & 10 & 0 & 0 & 0 & $\begin{array}{l}8 \\
8 \\
8 \\
8 \\
0 \\
0\end{array}$ & $\begin{array}{l}8 \\
8 \\
8 \\
8 \\
\text { वे }\end{array}$ \\
\hline & $\begin{array}{l}\text { 옳 } \\
\text { 을 } \\
\text { 듞 } \\
\text { J }\end{array}$ & - & - & 0 & 0 & $\leftarrow$ & $\sim$ & $\sim$ & - & $m$ & - & 0 & 0 & 0 & 0 & 0 & $\sim \sim$ & 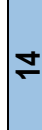 \\
\hline \multicolumn{2}{|c|}{ 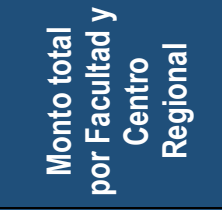 } & 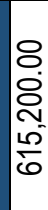 & 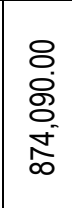 & $\begin{array}{l}8 \\
8 \\
8 \\
8 \\
8\end{array}$ & 0 & $\begin{array}{l}8 \\
8 \\
8 \\
\frac{0}{N} \\
\stackrel{1}{N}\end{array}$ & $\begin{array}{l}8 \\
8 \\
8 \\
8 \\
\text { ஜ్ }\end{array}$ & $\begin{array}{l}8 \\
8 \\
8 \\
8 \\
\circ\end{array}$ & 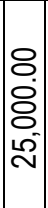 & $\mid \begin{array}{l}8 \\
8 \\
8 \\
8 \\
15 \\
15 \\
1\end{array}$ & $\begin{array}{l}8 \\
8 \\
8 \\
0 \\
\text { م }\end{array}$ & $\begin{array}{l}8 \\
8 \\
8 \\
8 \\
10 \\
\\
\end{array}$ & 8 & $\begin{array}{l}8 \\
8 \\
8 \\
8 \\
8 \\
\infty \\
\infty\end{array}$ & 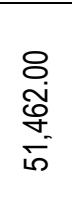 & 8. & 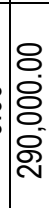 & $\begin{array}{l}8 \\
\text { กิ } \\
\frac{\pi}{\infty} \\
\frac{\infty}{\infty}\end{array}$ \\
\hline \multicolumn{2}{|c|}{ 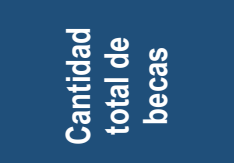 } & 10 & $\stackrel{\sim}{\sim}$ & $m$ & 0 & 0 & $\simeq$ & $\sim$ & - & $\nabla$ & $\sigma$ & $\nabla$ & ' & 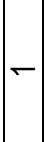 & 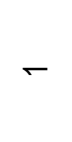 & ' & م & $\infty$ \\
\hline \multicolumn{2}{|c|}{ 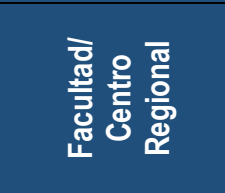 } & $\frac{\pi}{\frac{\pi}{0}}$ & 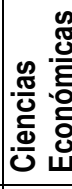 & 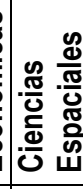 & 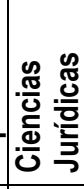 & 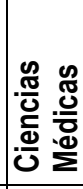 & 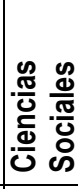 & 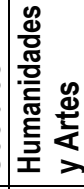 & 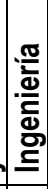 & $\mid \begin{array}{l}\frac{\pi}{5} \\
\text { 응 } \\
\text { 끔 } \\
\text { 응 }\end{array}$ & 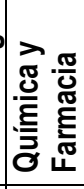 & Uي & $\frac{\overleftarrow{\zeta}}{\not{\underline{\varphi}}}$ & O্ & 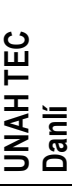 & $\frac{\infty}{3}$ & \multirow{2}{*}{\multicolumn{2}{|c|}{$\frac{2}{5}$}} \\
\hline \multicolumn{2}{|c|}{$\stackrel{\circ}{z}$} & - & ๙ & $m$ & $\nabla$ & ? & 6 & $\sim$ & $\infty$ & 0 & 우 & $F$ & $\cong$ & $m$ & 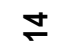 & n) & 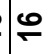 & \\
\hline
\end{tabular}

Fuente: La investigación científica en la UNAH, Todo en cifras 2007-2015. 
Cuadro 4. UNAH 2014-2016: Total de becas postuladas y total de becas de estudiantes de grado y posgrado

\begin{tabular}{|c|c|c|c|}
\hline \multirow{2}{*}{ Año } & \multicolumn{3}{|c|}{ Total de becas postuladas } \\
\cline { 2 - 4 } & General & De estudiantes de grado y posgrado & $\%$ \\
\hline 2014 & 116 & 45 & 38.8 \\
\hline 2015 & 95 & 28 & 29.5 \\
\hline 2016 & 113 & 40 & 35.4 \\
\hline Total & 324 & 113 & 34.9 \\
\hline
\end{tabular}

Fuente: La investigación científica en la UNAH, Todo en cifras 2007-2015.

Como puede observarse en los Cuadros 4 y 5 , el total de becas postuladas entre los años 2014-2016 fueron 324, de las que 32 provinieron de estudiantes de grado $(9.9 \%)$ y 81 de estudiantes de posgrado (25\%). El porcentaje de aprobación a sus solicitudes en el conjunto de esos tres años fue de $43.8 \%$ en los estudiantes de grado y de $61.7 \%$ en los estudiantes de posgrado, y $56.6 \%$ para ambos. En las becas de grado se observa un aumento en la aprobación de 2014 a 2015 y después una disminución de 2015 a 2016; en cambio en los estudiantes de posgrado la tendencia es al revés: de 2014 a 2015 disminuye el porcentaje de aprobación, mientras que aumenta entre 2015 y 2016 . Se considera que este incremento se debió al apoyo que gestionaron de asesoría metodológica. Es decir, no se observa una tendencia uniforme para estos dos tipos de becas entre 2014 y 2016.

Cuadro 5. UNAH 2014-2016: Becas de estudiantes de grado y posgrado, postuladas y aprobadas

\begin{tabular}{|c|c|c|c|c|c|c|c|c|c|}
\hline \multirow[b]{2}{*}{ Año } & \multicolumn{3}{|c|}{$\begin{array}{c}\text { Total Becas de estudiantes } \\
\text { de grado y posgrado }\end{array}$} & \multicolumn{3}{|c|}{$\begin{array}{c}\text { Becas de estudiantes } \\
\text { de grado }\end{array}$} & \multicolumn{3}{|c|}{$\begin{array}{c}\text { Becas de estudiantes de } \\
\text { posgrado }\end{array}$} \\
\hline & $\begin{array}{l}\frac{9}{0} \\
\frac{\pi}{0} \\
\frac{0}{5} \\
\frac{0}{0} \\
\stackrel{0}{0}\end{array}$ & 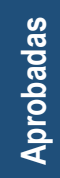 & $\%$ & 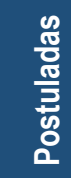 & $\begin{array}{l}\frac{\infty}{d} \\
\frac{0}{0} \\
\frac{0}{0} \\
\frac{0}{2} \\
\frac{0}{4}\end{array}$ & $\%$ & 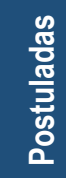 & 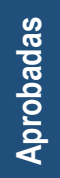 & $\%$ \\
\hline 2014 & 45 & 27 & 60.0 & 6 & 1 & 16.7 & 39 & 26 & 66.7 \\
\hline 2015 & 28 & 10 & 35.7 & 8 & 5 & 62.5 & 20 & 5 & 25.0 \\
\hline 2016 & 40 & 25 & 62.5 & 18 & 8 & 44.4 & 22 & 17 & 77.3 \\
\hline Total & 113 & 64 & 56.6 & 32 & 14 & 43.8 & 81 & 50 & 61.7 \\
\hline
\end{tabular}

Fuente: Dirección de Investigación Científica, UNAH 2016 


\section{Fortalezas y debilidades en las solicitudes de estudiantes}

Al analizar los proyectos de investigación entre los años 2014 al 2016, se observa que las debilidades más relevantes son, en primer lugar, la metodología aplicada, luego la presentación de la matriz de operacionalización de variables y/o la matriz de categorización de variables, según sea el alcance del estudio y el marco teórico utilizado. En la metodología, las debilidades más recurrentes son la no determinación del enfoque, así como la no relación entre el enfoque elegido y las técnicas a utilizar. La mayoría de las propuestas no presentan las matrices o son presentadas de forma incorrecta. Con relación al marco teórico, éste debe contener el fundamento teórico en que se estructura la investigación, lo que no siempre es proporcionado.

En cuanto a la operacionalización de variables, en las solicitudes recibidas se observan debilidades tanto en la determinación de variables -dependientes e independientes-, así como también en diferenciar aquellas que pueden distorsionar el análisis. También hay limitaciones en desglosar las variables intervinientes en los sectores que participan en su determinación y, especialmente, en buscar los indicadores que permitan medirlas.

\section{CONCLUSIONES Y RECOMENDACIONES}

La política de investigación de la UNAH va dirigida a desarrollar la investigación en la institución, considerándose prioritario tanto entre los estudiantes de grado y posgrado así como entre los profesores. Este artículo se centró en identificar la participación de los estudiantes en becas de investigación. Del total de 233 becas de investigación otorgadas a profesores y estudiantes entre el año 2007 hasta el 2015, el 26.6\% del total de becas fueron otorgadas a estudiantes de grado y posgrado.

En la universidad la incursión de estudiantes de ambos niveles en las becas de investigación científica está dando pasos importantes. Hay un incremento en la participación en investigaciones en los últimos años en ellos, así como también en el involucramiento de la mayoría de Facultades y Centros Regionales; denotando mayor participación, especialmente en las facultades de Ciencias Económicas, Ciencias y Ciencias Sociales, que representan $65.4 \%$ de becas con respecto al total de becas obtenidas por los estudiantes.

En cuanto a la relación de participación de los estudiantes de grado y posgrado que 
postularon para obtener beca de investigación con respecto al promedio de estudiantes matriculados en su último año en la UNAH en el período 2014-2016, se observa que en grado se obtuvo un promedio de 1.1 estudiantes por cada mil matriculados en el período mencionado. En el caso de los estudiantes de posgrado la participación total -como se esperaría- es mayor: participó un total de 2.4 por cada 100 estudiantes de posgrado matriculados en el período 2014-2016.

Al analizar el porcentaje de participación de los estudiantes con respecto al total de becas se observa que en 2014 fue de $38.8 \%$, en 2015 del 29.5\% y en 2016 de $35.4 \%$ con un promedio durante los tres años de $34.9 \%$, es decir un poco más de la tercera parte del total de becas postuladas fueron de estudiantes.

El total general de becas postuladas entre los años 2014-2016 fueron 324, de las cuales 32 provinieron de estudiantes de grado y 81 de estudiantes de posgrado. El porcentaje de aprobación a sus solicitudes en los años mencionados fue de $43.8 \%$ en los estudiantes de grado y de $61.7 \%$ en los estudiantes de posgrado. El $4.3 \%$ del total de los fondos otorgados a becas de investigación se destinó a becas de estudiantes de grado y posgrado, lo que equivale a un monto de L. 1,724,090.00. Si se agrega la participación de profesores con becas de docentes egresados de posgrado -utilizadas para hacer tesis de graduación en los posgrados que cursan -se observa que el monto casi se duplica, al pasar a $L 3,178,752.00$ con respecto a si sólo se toman los estudiantes de grado y posgrado.

Por facultades se observan que lideran las facultades de Ciencias Económicas, Ciencias y Ciencias Sociales con $61.4 \%$ del monto total destinado para investigación de los estudiantes. Es importante la participación de los estudiantes en investigaciones pues permite una formación profesional más sólida así como una percepción crítica de la respectiva realidad que analizan, pues para realizar investigación el individuo se ha detenido a detectar los vacíos teóricos o los problemas que aquejan la sociedad y, con ello, se logra contribuir al desarrollo científico y tecnológico y a la resolución de los problemas del país. 


\section{BIBLIOGRAFÍA}

Dirección de Investigación Científica, U. (2015). Compendios de Investigación Científica, Serie 1.

Dirección de Investigación Científica, U. (2015). Compendios de Investigación Científica, Serie 3. Tegucigalpa.

Maldonado, L. (2007). Universidad Católica de Colombia. Recuperado el 01 de Diciembre de 2016, de Universidad Católica de Colombia: http://repository.ucatolica.edu.co:8080/bitstream/10983/502/1/Stud_2-2_A05_MALDONADO- LANDAZABAL.pdf 
